Article

\title{
Low Detection Rate of H3K27M Mutations in Cerebrospinal Fluid Obtained from Lumbar Puncture in Newly Diagnosed Diffuse Midline Gliomas
}

\author{
Jotaro On ${ }^{1} \mathbb{D}^{D}$, Manabu Natsumeda ${ }^{1, *(\mathbb{D}}$, Jun Watanabe ${ }^{1} \mathbb{D}$, Shoji Saito ${ }^{1}$, Yu Kanemaru ${ }^{1}$, Hideaki Abe ${ }^{1}$, \\ Yoshihiro Tsukamoto $^{1}$, Masayasu Okada ${ }^{1} \mathbb{D}$, Makoto Oishi ${ }^{1}$, Junichi Yoshimura ${ }^{1}$, Akiyoshi Kakita ${ }^{2}$ \\ and Yukihiko Fujii ${ }^{1}$
}

Citation: On, J.; Natsumeda, M.; Watanabe, J.; Saito, S.; Kanemaru, Y.; Abe, H.; Tsukamoto, Y.; Okada, M.; Oishi, M.; Yoshimura, J.; et al. Low Detection Rate of H3K27M Mutations in Cerebrospinal Fluid Obtained from Lumbar Puncture in Newly Diagnosed Diffuse Midline Gliomas. Diagnostics 2021, 11, 681. https:// doi.org/10.3390/diagnostics11040681

Academic Editor:

Angela Mastronuzzi

Received: 2 March 2021

Accepted: 4 April 2021

Published: 9 April 2021

Publisher's Note: MDPI stays neutral with regard to jurisdictional claims in published maps and institutional affiliations.

Copyright: (c) 2021 by the authors. Licensee MDPI, Basel, Switzerland. This article is an open access article distributed under the terms and conditions of the Creative Commons Attribution (CC BY) license (https:// creativecommons.org/licenses/by/ $4.0 /)$.
1 Department of Neurosurgery, Brain Research Institute, Niigata University, Niigata 951-8585, Japan; jotaro-on_silver@sky.hi-ho.ne.jp (J.O.); watanabejun1003@yahoo.co.jp (J.W.); sho-2.s@bri.niigata-u.ac.jp (S.S.); yu.k93@bri.niigata-u.ac.jp (Y.K.); hideabe04161983@gmail.com (H.A.); yoshi.tsukamoto@me.com (Y.T.); masayasu_okd@bri.niigata-u.ac.jp (M.O.); mac.oishi@mac.com (M.O.); junyoshi@bri.niigata-u.ac.jp (J.Y.); yfujii@bri.niigata-u.ac.jp (Y.F.)

2 Department of Pathology, Brain Research Institute, Niigata University, Niigata 951-8585, Japan; kakita@bri.niigata-u.ac.jp

* Correspondence: natsumeda@bri.niigata-u.ac.jp

Abstract: Recent studies have suggested the feasibility of detecting H3K27M mutations in the cerebrospinal fluid of diffuse midline glioma (DMG) patients. However, cerebrospinal fluid from patients in these studies were collected mainly during biopsy, ventriculo-peritoneal shunt procedures or postmortem. We assessed circulating tumor DNA (ctDNA) extracted from cerebrospinal fluid (CSF) and plasma in a series of 12 radiographically suspected and/or pathologically confirmed diffuse midline glioma patients and assessed for H3F3A K27M mutation using digital droplet PCR. In 10 patients, CSF was obtained by lumbar puncture at presentation. A definitive detection of $H 3 F 3 A$ K27M mutation was achieved in only one case (10\%); H3F3A K27M mutation was suspected in three other cases (30\%). H3F3A K27M mutation was detected in two patients in CSF obtained by ventricular tap during a ventriculo-peritoneal shunt for obstructive hydrocephalus. Cases in which a definitive assessment was possible (definite $H 3 F 3 A \mathrm{~K} 27 \mathrm{M}$ or definite $H 3 F 3 A$ wildtype) tended to be younger (median 7.5 years vs. 40.5 years; $p=0.07$ ) and have a higher concentration of CSF protein (median $123 \mathrm{mg} / \mathrm{dL}$ vs. $27.5 \mathrm{mg} / \mathrm{dL} ; p=0.21$ ) compared to nondefinite cases. Low proliferation and apoptotic rates seemed to be characteristics of DMG unfavorable for liquid biopsy. More advanced lesions with necrosis and evidence of dissemination were unlikely to be candidates for lumbar puncture due to the fear of exacerbating obstructive hydrocephalus. Methods to safely sample CSF and a more sensitive detection of ctDNA are necessary for reliable liquid biopsy of DMG at presentation.

Keywords: liquid biopsy; H3K27M-mutant; diffuse midline glioma; circulating tumor DNA; diagnosis

\section{Introduction}

Recurrent HIST1H3B (H3.1K27M) or H3F3A (H3.3K27M) have been reported in 50 to $80 \%$ of diffuse intrinsic pontine gliomas and thalamic gliomas [1-6]; thus, the entity "diffuse midline gliomas, H3K27M-mutant" has been introduced in the revised fourth edition of World Health Organization Classification Tumours of the Central Nervous System (WHO2016) [7]. The discovery of these diagnostic, genetic abnormalities has led to excitement about noninvasive diagnosis by liquid biopsy of diffuse midline gliomas (DMGs), in which radical resection is often not possible due to localization to the brainstem and thalamus.

We have previously reported a $100 \%$ match of the MYD88 mutation status in circulating tumor DNA (ctDNA) extracted from cerebrospinal fluid (CSF) and biopsied tissue in 21 primary and secondary central nervous system lymphoma patients using droplet 
digital PCR (ddPCR) [8]. Recent papers have suggested the feasibility of detecting H3K27M mutations in CSF by ddPCR $[9,10]$ or next generation sequencing panel analysis [11] of ctDNA, giving the impression that a liquid biopsy for the diagnostic detection of H3K27M mutations in DMGs is feasible before the initiation of radiation treatment. However, a closer look at the papers reveals that CSF collected from patients in these studies mainly occurred during biopsy, ventriculo-peritoneal shunting or postmortem. In the present study, we shed light on the prospects of liquid biopsy of DMG at presentation and discuss what characteristics of brain tumors make the reliable detection of ctDNA in CSF possible.

\section{Materials and Methods}

Twelve patients with radiographically suspected diffuse midline glioma, treated at the Department of Neurosurgery, Niigata University Hospital between November 2017 and January 2021, who underwent liquid biopsy, were enrolled in the study. Written consent was obtained from patients and families for liquid biopsy after approval from the institutional review board (\#G2018-0008). Detailed methods of ddPCR have been previously published [8]. Briefly, $1 \mathrm{~mL}$ of cerebrospinal fluid was obtained and centrifuged at $4{ }^{\circ} \mathrm{C}$, $1000 \times g$ for 10 minutes to remove cells, and the supernatant was stored at $-80^{\circ} \mathrm{C}$ within 30 minutes of collection. ctDNA was extracted using the Maxwell RSC ccfDNA Plasma Kit (RSC; Promega, Leiden, The Netherlands), according to the manufacturer's instructions. For all samples, DNA was dissolved in $60 \mu \mathrm{L}$ of elution buffer and stored at $-20^{\circ} \mathrm{C}$ until further use. ddPCR reagents and primer/probe mix for $H 3 F 3 A$ K27M were purchased from Bio-Rad (Hercules, CA, USA). A $20 \mu \mathrm{L}$ PCR mix, composed of $10 \mu \mathrm{L} 2 \times$ ddPCR Supermix for Probes (no deoxyuridine triphosphate; Bio-Rad), $1 \mu \mathrm{L}$ ddPCR Mutation Assay (Bio-Rad) and $9 \mu \mathrm{L}$ ctDNA, was loaded into sample wells of an eight-channel disposable droplet generator cartridge (Bio-Rad). An additional $70 \mu \mathrm{L}$ of droplet generation oil (Bio-Rad) was loaded into the oil well for each channel. After droplet generation, the droplets were transferred into a 96-well PCR plate and subjected to thermal cycling. Amplification was carried out on the $20 \mu \mathrm{L}$ reaction mixture on the QX-200 ddPCR system (Bio-Rad). After PCR, the 96-well PCR plate was subjected to the QX-200 droplet reader (Bio-Rad), and data were analyzed by QuantaSoft analysis software (Bio-Rad). H3F3A K27M-mutation specific signals were generated in the hexachloro-fluorescein channel. We considered definite mutant cases to have a fractional abundance of $0.1 \%$ or more and to have three or more mutant droplets and/or wildtype droplets detected. Cases in which only one or two mutant droplets were detected were considered H3K27M-mutant-suspect. Cases where two or fewer wildtype droplets were detected were considered unreliable. ddPCR was performed in at least two separate wells for each case whenever possible. Surgical removal or biopsy was performed in two cases (cases \#7 and \#11) with genetic confirmation of H3F3A K27M.

Comparisons between two groups were carried out using the Mann-Whitney $U$ test and tests for associations between different parameters by the Fisher's exact test for $2 \times 2$ contingency tables. Statistical analyses were performed using GraphPad Prism 9 software (GraphPad Software, http:/ / www.graphpad.com accessed on 6 April 2021). $p<0.05$ was considered significant.

\section{Results}

\subsection{Patient Characteristics}

Lesions were located at the pons in eight patients, medulla oblongata in one patient, thalamus in two patients and temporal lobe in one patient (Table 1). A liquid biopsy was performed a total of 19 times in 12 patients: at presentation in 10 patients, during adjuvant treatment in one patient, at recurrence in three patients and during ventriculoperitoneal shunting in four patients. In one patient, a liquid biopsy was performed at presentation using both CSF and plasma. CSF was obtained by lumbar puncture in 10 patients, ventricular tap in three patients, and plasma was obtained from four patients. Pre- and post-contrast MR imaging was performed in all patients. Contrast enhancement 
was observed in 10 patients at presentation, and in one patient contrast enhancement was not observed at presentation but at relapse. Necrosis was observed in three patients at presentation and in one patient at relapse. Evidence of leptomeningeal disease was observed in three patients.

Table 1. Summary of patient and imaging characteristics.

\begin{tabular}{|c|c|c|c|c|c|c|c|c|c|c|}
\hline Case\# & Age & Sex & Location & $\begin{array}{c}\text { Timing of } \\
\text { Liquid Biopsy }\end{array}$ & $\begin{array}{l}\text { Type of } \\
\text { Fluid }\end{array}$ & $\begin{array}{c}\text { CSF } \\
\text { Source }\end{array}$ & Assessment & $\begin{array}{c}\text { Gd- } \\
\text { Enhancement }\end{array}$ & Necrosis & Dissemination \\
\hline 1 & 7 & $\mathrm{~F}$ & Pons & At presentation & CSF & $\mathrm{LP}^{1}$ & H3К $27 \mathrm{M}$ & + & + & + \\
\hline 2 & 5 & $\mathrm{~F}$ & Pons & At presentation & CSF & $\mathrm{LP}^{1}$ & $\mathrm{WT}^{2}$ & + & + & - \\
\hline 3 & 59 & $\mathrm{M}$ & Pons & At presentation & CSF & $\mathrm{LP}^{1}$ & $\mathrm{WT}^{2}$ & + & - & - \\
\hline \multirow[t]{2}{*}{4} & 9 & M & Pons & At presentation & CSF & $\mathrm{LP}^{1}$ & $\mathrm{WT}^{2}$ & \pm & - & - \\
\hline & & & & At presentation & Plasma & $\mathrm{N} / \mathrm{A}^{3}$ & H3K27M? ${ }^{4}$ & \pm & - & - \\
\hline 5 & 31 & $\mathrm{~F}$ & Pons & At presentation & CSF & $\mathrm{LP}^{1}$ & H3K27M? ${ }^{4}$ & \pm & - & + \\
\hline \multirow[t]{2}{*}{6} & 64 & $\mathrm{M}$ & Pons & At presentation & CSF & $\mathrm{LP}^{1}$ & H3K27M? ${ }^{4}$ & - & - & - \\
\hline & & & & At recurrence & CSF & $\mathrm{LP}^{1}$ & $\mathrm{H} 3 \mathrm{~K} 27 \mathrm{M} ?^{4}$ & + & \pm & - \\
\hline \multirow[t]{3}{*}{7} & 23 & $\mathrm{M}$ & $\mathrm{T}^{5}$ & At presentation & $\mathrm{CSF}$ & LP 1 & H3K27M? ${ }^{4}$ & - & - & - \\
\hline & & & & At recurrence & Plasma & $\mathrm{N} / \mathrm{A}^{3}$ & H3K27M? ${ }^{4}$ & - & - & - \\
\hline & & & & During VP shunt & CSF & Vent tap ${ }^{6}$ & $\mathrm{H} 3 \mathrm{~K} 27 \mathrm{M} ?^{4}$ & - & - & - \\
\hline 8 & 69 & M & Thal $^{7}$ & At presentation & CSF & $\mathrm{LP}^{1}$ & Unreliable & + & - & - \\
\hline \multirow[t]{2}{*}{9} & 7 & $\mathrm{~F}$ & Pons & At presentation & CSF & $\mathrm{LP}^{1}$ & Unreliable & + & + & - \\
\hline & & & & During VP shunt & CSF & Vent tap ${ }^{6}$ & Unreliable & + & + & - \\
\hline 10 & 50 & $\mathrm{~F}$ & $\mathrm{MO}^{8}$ & At presentation & CSF & LP 1 & Unreliable & + & - & - \\
\hline \multirow[t]{2}{*}{11} & 6 & $\mathrm{~F}$ & Thal $^{7}$ & During VP shunt & CSF & Vent tap ${ }^{6}$ & H3K27M & + & - & + \\
\hline & & & & At recurrence & Plasma & $\mathrm{N} / \mathrm{A}^{3}$ & H3K27M? ${ }^{4}$ & + & - & + \\
\hline \multirow[t]{2}{*}{12} & 8 & $\mathrm{~F}$ & Pons & During treatment & Plasma & $\mathrm{N} / \mathrm{A}^{3}$ & $\mathrm{WT}^{2}$ & + & - & - \\
\hline & & & & At recurrence & Plasma & $\mathrm{N} / \mathrm{A}^{3}$ & H3К27M & + & - & - \\
\hline
\end{tabular}

${ }^{1}$ Lumbar puncture, ${ }^{2}$ Wildtype, ${ }^{3}$ Not applicable, ${ }^{4}$ H3K27M suspect, ${ }^{5}$ Temporal lobe, ${ }^{6}$ Ventricular tap, ${ }^{7}$ Thalamus, ${ }^{8}$ Medulla oblongata.

\subsection{Detection Rate of H3F3A K27M at Presentation}

Lumbar puncture was performed in 10 patients at presentation (Table 1). However, a definite detection of the H3F3A K27M mutation was obtained in only one (10\%) patient showing diffuse hyperintensity of the pons on T2-weighted MR images (Figure 1A) and an exophytic growth at the lower pons with encasement of vertebral arteries by enhancing tumor on post-contrast images (Figure 1B). A high variant allele frequency (VAF) of $76.6 \%$ was detected (Figure 1C). Exophytic growth at the lower pons and encasement of the vertebral arteries but not hydrocephalus was observed in this case. H3F3A K27M was suspected in three $(30 \%)$ other patients at presentation, but only one or two mutant droplets were detected from each well in these patients (Table 2). Two cases were wildtype for H3F3A, in which wildtype droplets but not mutant droplets were observed. In three (30\%) cases, the ddPCR results were considered unreliable, with a sufficient number of mutant and wildtype droplets not being detected.

\subsection{Detection Rate of H3F3A K27M at Recurrence}

A definitive detection of $H 3 F 3 A$ K27M was obtained at recurrence from two patients, both in CSF obtained from ventricular tap during ventriculo-peritoneal shunting for obstructive hydrocephalus in a recurrent DMG case with heterogeneously enhancing residual lesion of the right thalamus (Figure 2A) and enlarged ventricles (Figure 2B). Multiple H3F3A K27M-mutant droplets were detected by ddPCR analysis (Figure 2C). However, a definitive detection of $H 3 F 3 A$ K27M was not achieved in patients undergoing lumbar puncture at recurrence in a DMG patient showing a heterogeneously enhancing lesion with possible necrosis (Figure 3A) and perifocal hyperintensity on fluid attenuated inversion recovery (FLAIR) (Figure 3B). Since mutant droplets were detected from both wells by ddPCR analysis, H3F3A K27M mutation was strongly considered (Figure 3C,D). 


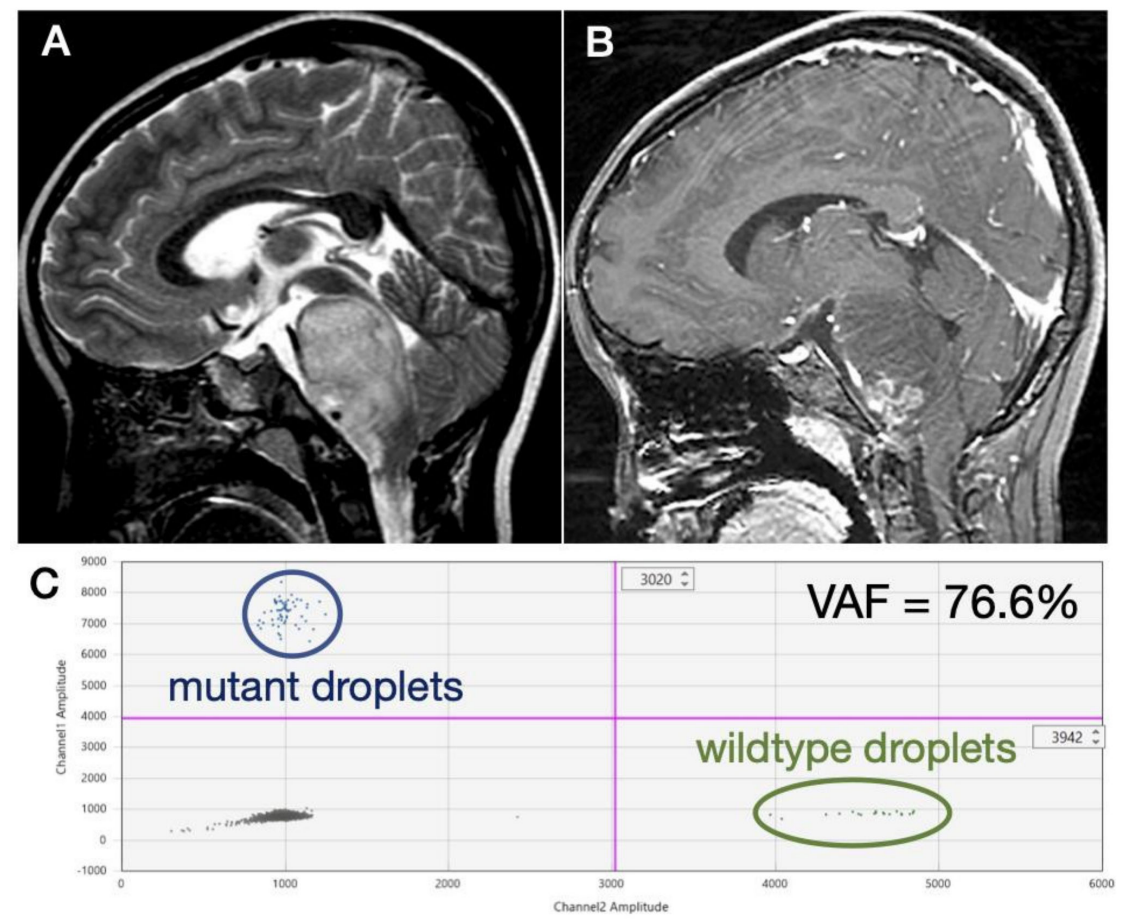

Figure 1. Representative case in which the H3F3A mutation was detected in CSF obtained by lumbar puncture at presentation. MR images show (A) a diffuse hyperintensity of the pons on T2-weighted images and (B) an exophytic growth at the lower pons and encasement of vertebral arteries by enhancing tumor on post-contrast images. (C) Many mutant and wildtype droplets were detected, and the variant allele frequency was $76.6 \%$.

Table 2. Summary of liquid biopsies.

\begin{tabular}{|c|c|c|c|c|c|c|c|c|}
\hline Case\# & $\begin{array}{l}\text { Type of } \\
\text { Fluid }\end{array}$ & $\begin{array}{l}\text { CSF } \\
\text { Source }\end{array}$ & $\begin{array}{l}\text { DNA Conc } \\
\text { (ng/mL) }\end{array}$ & $\begin{array}{c}\text { Protein } \\
\text { Conc } \\
\text { (mg/dL) }\end{array}$ & $\begin{array}{c}\text { CSF } \\
\text { Cytology } \\
\text { Class }\end{array}$ & $\begin{array}{l}\text { Mut/WT1 } \\
\text { VAF1(\%) }\end{array}$ & $\begin{array}{l}\text { Mut/WT2 } \\
\text { VAF2(\%) }\end{array}$ & Assessment \\
\hline 1 & CSF & $\mathrm{LP}^{1}$ & 228 & 69 & I & $62 / 19(76.6)$ & $50 / 12(80.7)$ & H3K27M \\
\hline 2 & CSF & $\mathrm{LP}^{1}$ & 186 & 14 & I & $0 / 15(0.0)$ & $0 / 4(0.0)$ & $\mathrm{WT}^{2}$ \\
\hline 3 & CSF & $\mathrm{LP}^{1}$ & 216 & 123 & I & $0 / 135(0.0)$ & $0 / 185(0.0)$ & $\mathrm{WT}^{2}$ \\
\hline \multirow[t]{2}{*}{4} & CSF & $\mathrm{LP}^{1}$ & 432 & 217 & I & $0 / 22(0.0)$ & $\mathrm{N} / \mathrm{A}^{3}$ & $\mathrm{WT}^{2}$ \\
\hline & Plasma & $\mathrm{N} / \mathrm{A}^{3}$ & 468 & $\mathrm{~N} / \mathrm{A}^{3}$ & $\mathrm{~N} / \mathrm{A}^{3}$ & $2 / 69(2.8)$ & $\mathrm{N} / \mathrm{A}^{3}$ & H3К27M? ${ }^{4}$ \\
\hline 5 & CSF & $\mathrm{LP}^{1}$ & 264 & 19 & $\mathrm{I}$ & $0 / 7(0.0)$ & $1 / 21(4.5)$ & H3K27M? ${ }^{4}$ \\
\hline \multirow[t]{2}{*}{6} & CSF & $\mathrm{LP}^{1}$ & 216 & 32 & I & $1 / 0(100)$ & $2 / 1(66.7)$ & H3K27M? ${ }^{4}$ \\
\hline & CSF & $\mathrm{LP}^{1}$ & 318 & 69 & I & $2 / 3(40.0)$ & $1 / 5(16.7)$ & H3K27M? ${ }^{4}$ \\
\hline \multirow[t]{3}{*}{7} & CSF & $\mathrm{LP}^{1}$ & 504 & $\mathrm{~N} / \mathrm{A}^{3}$ & $\mathrm{~N} / \mathrm{A}^{3}$ & $1 / 39(2.5)$ & $\mathrm{N} / \mathrm{A}^{3}$ & $\mathrm{H} 3 \mathrm{~K} 27 \mathrm{M} ?^{4}$ \\
\hline & Plasma & $\mathrm{N} / \mathrm{A}^{5}$ & 366 & $\mathrm{~N} / \mathrm{A}^{3}$ & $\mathrm{~N} / \mathrm{A}^{3}$ & $1 / 37$ (2.6) & $\mathrm{N} / \mathrm{A}^{3}$ & H3K27M? ${ }^{4}$ \\
\hline & CSF & Vent tap 5 & 90 & $\mathrm{~N} / \mathrm{A}^{3}$ & $\mathrm{~N} / \mathrm{A}^{3}$ & 2/12 (14.3) & $1 / 9(10.0)$ & H3К27M? ${ }^{4}$ \\
\hline 8 & CSF & $\mathrm{LP}^{1}$ & 216 & $\mathrm{~N} / \mathrm{A}^{3}$ & $\mathrm{~N} / \mathrm{A}^{3}$ & $0 / 0(0.0)$ & $0 / 0(0.0)$ & Unreliable \\
\hline \multirow[t]{2}{*}{9} & CSF & $\mathrm{LP}^{1}$ & 330 & 23 & I & $0 / 1(0.0)$ & $0 / 0(0.0)$ & Unreliable \\
\hline & CSF & Vent tap 5 & 306 & $\mathrm{~N} / \mathrm{A}^{3}$ & $\mathrm{~N} / \mathrm{A}^{3}$ & $0 / 0(0.0)$ & $0 / 0(0.0)$ & Unreliable \\
\hline 10 & CSF & $\mathrm{LP}^{1}$ & 120 & 29 & I & $0 / 2(0.0)$ & $0 / 0(0.0)$ & Unreliable \\
\hline 11 & CSF & Vent tap 5 & 330 & 538 & III & $\begin{array}{c}151 / 173 \\
(46.6)\end{array}$ & $\begin{array}{c}120 / 165 \\
(42.0)\end{array}$ & H3К27M \\
\hline \multirow{3}{*}{12} & Plasma & $\mathrm{N} / \mathrm{A}^{3}$ & 294 & $\mathrm{~N} / \mathrm{A}^{3}$ & $\mathrm{~N} / \mathrm{A}^{3}$ & $0 / 14(0.0)$ & $1 / 36(2.8)$ & H3K27M? ${ }^{4}$ \\
\hline & Plasma & $\mathrm{N} / \mathrm{A}^{3}$ & 414 & $\mathrm{~N} / \mathrm{A}^{3}$ & $\mathrm{~N} / \mathrm{A}^{3}$ & $0 / 50(0.0)$ & $\mathrm{N} / \mathrm{A}^{3}$ & $\mathrm{WT}^{2}$ \\
\hline & CSF & Vent tap 5 & 222 & $\mathrm{~N} / \mathrm{A}^{3}$ & $\mathrm{~N} / \mathrm{A}^{3}$ & $22 / 30(42.3)$ & $30 / 40(42.9)$ & H3К27M \\
\hline
\end{tabular}

${ }^{1}$ Lumbar puncture, ${ }^{2}$ Wildtype, ${ }^{3}$ Not applicable, ${ }^{4}$ H3K27M suspect, ${ }^{5}$ Ventricular tap. 

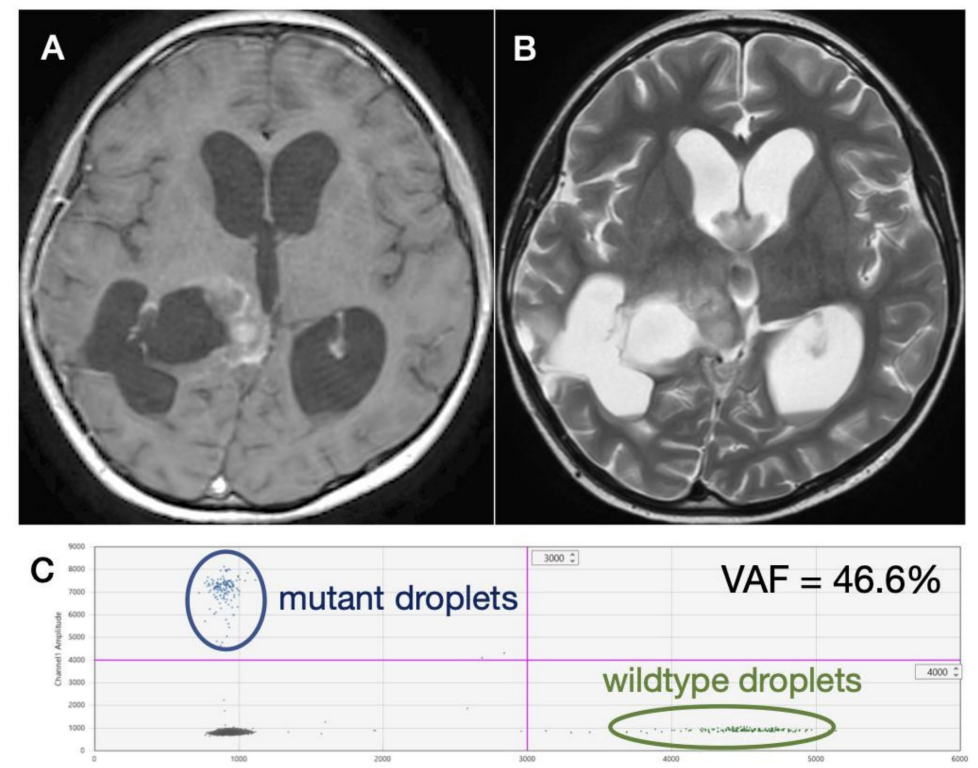

Figure 2. A recurrent DMG case in which H3F3A K27M was detected in CSF obtained during ventriculo-peritoneal shunting. MR images show (A) a heterogeneously enhancing residual lesion of the right thalamus and (B) enlarged ventricles. (C) Multiple H3F3A K27M-mutant droplets were detected by ddPCR analysis.
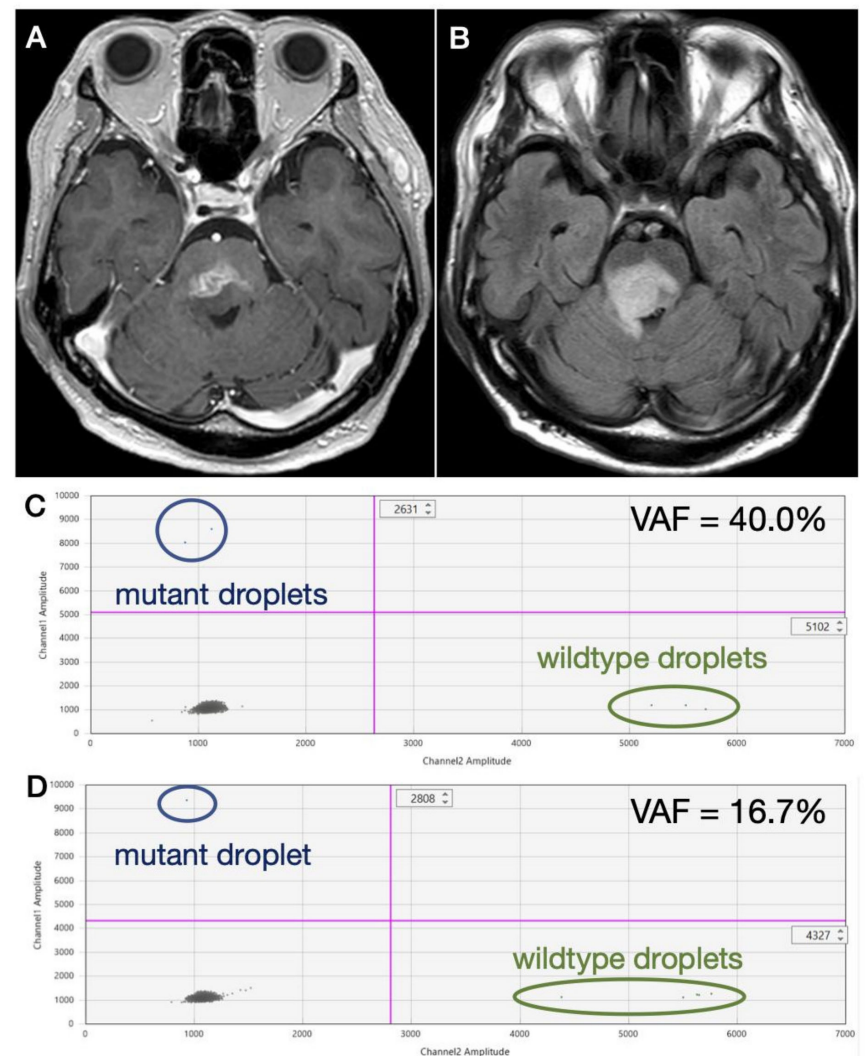

Figure 3. A recurrent DMG case in which $H 3 F 3 A$ K27M was suspected. MR images show (A) a heterogeneously enhancing lesion with possible necrosis and (B) perifocal hyperintensity on fluid attenuated inversion recovery (FLAIR). Since mutant droplets were detected from both wells by ddPCR analysis, H3F3A K27M mutation was strongly considered. However, since only (C) two and (D) one mutant droplets were detected from each well, a definitive diagnosis was not achieved. 


\subsection{Comparison of Definite versus Nondefinite Cases}

We compared the clinical, radiographical and CSF characteristics of cases in which there was a definite diagnosis (defined as either a definite H3F3A K27M mutation or definite H3F3A K27 wildtype) versus nondefinite (defined as H3F3A K27M-suspect or unreliable) cases (Table 3). Due to the lack of sample size, none of the analyzed factors yielded significant $p$-values, but there was a tendency for definite cases to be younger (median 7.5 years vs. 40.5 years; $p=0.07$ ) and to have a higher concentration of CSF protein (median $123 \mathrm{mg} / \mathrm{dL}$ vs. $27.5 \mathrm{mg} / \mathrm{dL} ; p=0.21$ ).

Table 3. Characteristics of definite vs. nondefinite cases.

\begin{tabular}{cccc}
\hline Characteristic & Definite & Non-Definite & $p$-Value \\
\hline Age (y); median (range) & $(n=6)$ & $(n=6)$ & \\
Sex & $7.5(5-59)$ & $40.5(7-69)$ & 0.07 \\
Male; number (\%) & & \\
Female; number (\%) & $2(33)$ & $3(50)$ & $>0.99$ \\
Location & $4(67)$ & $3(50)$ & \\
Pons; number (\%) & $5(83)$ & $3(50)$ & 0.55 \\
Other; number (\%) & $1(17)$ & $3(50)$ & \\
CSF characteristics & & & \\
Protein; median (range) & $123(14-538)$ & $27.5(19-69)$ & $>0.99$ \\
Positive cytology; number (\%) & $1(17)$ & $0(0)$ & 0.45 \\
Radiographical features & & $4(67)$ & $>0.99$ \\
Gd-enhancement; number (\%) & $6(100)$ & $1(17)$ & $>0.99$ \\
Necrosis; number (\%) & $2(33)$ & $1(17)$ & \\
\hline Leptomeningeal disease: number (\%) & $2(33)$ &
\end{tabular}

\section{Discussion}

Recurrent HIST1H3B (H3.1K27M) or H3F3A (H3.3K27M) mutations have been reported in 50 to $80 \%$ of diffuse intrinsic pontine gliomas and thalamic gliomas [1-6]. Thus, the noninvasive diagnosis of DMGs through the detection of circulating H3K27M mutations in CSF and plasma at presentation is highly anticipated.

Three previous reports highlight the feasibility of detecting H3K27M mutations in DMG patients by ctDNA analysis in CSF and plasma [9-11]. However, taking a closer look at the results reveals that the detection of H3K27M mutations in CSF obtained by lumbar puncture at presentation is still highly challenging. Huang et al. detected HIST1H3B K27M mutations in four out of six $(66.7 \%)$ diffuse midline gliomas. In that study, CSF was either obtained from the ventricles by external ventricular drainage at tumor biopsy $(n=2)$, at reservoir placement $(n=2)$ or by reservoir tap during treatment $(n=2)$. Thus, CSF was not collected by lumbar tap before treatment in any of the cases. Subsequently, two large studies were reported. Pan et al. reported the detection of H3F3A K27M mutations $(n=27)$ or HIST1H3B K27M mutations $(n=5)$ in 32 out of $57(56 \%)$ DMG patients by next generation sequencing (NGS). CSF was obtained from the cisterns during biopsy in $52(91.2 \%)$ cases, from during ventriculo-peritoneal shunt procedures in two (3.5\%) cases, and by lumbar puncture in only three (5.7\%) cases [11]. Finally, Nazarian's group found H3K27M mutations in 42 out of $48(88 \%)$ subjects with DMGs. CSF was obtained from 28 patients, postmortem in 22 patients $(78.5 \%)$ and upfront in only four patients $(14.2 \%)[10]$.

An important study by Miller et al. detected ctDNA in CSF obtained by lumbar puncture in 42 out of $85(49.5 \%)$ patients harboring WHO grade 2, 3 and 4 gliomas using NGS. ctDNA was detected from a higher percentage of grade 4 tumors ( $64 \%$ to $44 \%)$, in patients with a positive CSF cytology ( $18 \%$ vs. $0 \%)$, higher CSF protein concentration (72 vs. 56), higher number of prior resections (two vs. one), in recurrent cases (55\% vs. $19 \%$ ) and in larger-volume tumors $\left(1553 \mathrm{~mm}^{2}\right.$ vs. $\left.373 \mathrm{~mm}^{2}\right)$ [12]. Additionally, ctDNA 
has been detected from plasma in $55 \%$ of glioblastoma patients using the Guardant $360^{\mathrm{R}}$ technology [13].

We have previously reported a high detection rate of MYD88 L265P mutations in CSF in primary and secondary CNS lymphoma patients using ddPCR, with a $100 \%$ match of the MYD88 L265P mutation status between tumor and CSF in 21 cases with available tumor samples [8]. A high VAF was detected in the majority of cases. By immediately centrifuging to remove cells and storing in $-80{ }^{\circ} \mathrm{C}$ after obtaining CSF and other body fluids, we were even able to detect MYD88 L265P mutations from the plasma and urine of PCNSL patients [14]. Since ctDNA is shed from cells undergoing apoptosis, tumors such as lymphomas with a rapid turnover may be easier to detect. The presence of lymphoglandular bodies, which are considered to reflect single cell apoptosis, is characteristic for PCNSL. Furthermore, a high lactate/lipid peak is often observed by magnetic resonance spectroscopy in PCNSL [15], reflecting rapid apoptosis. Furthermore, a marked contrast enhancement by Gadolinium, deep-seated location and frequent spinal dissemination are all features of PCNSL that favor the reliable detection of ctDNA. On the other hand, contrary to their dismal prognoses and midline location [16], DMGs are known to be slow growing $[17,18]$, frequently show only a subtle contrast enhancement, and often lack leptomeningeal disease and necrosis at presentation [19].

The limitations of the present study include the small sample size, low number of pathologically confirmed cases and inability to detect HIST1H3B K27M mutations [3] due to the lack of a reliable ddPCR primer. We have previously employed the same methods in PCNSL and achieved a high detection rate of MYD88 L265P and a 100\% match of the MYD88 mutation status between CSF and tumor. Therefore, we can safely say that, at present, detecting H3F3A K27M in CSF obtained by lumbar puncture in DMG patients is difficult. Using the Maxwell RSC ccfDNA Plasma Kit, up to $4 \mathrm{~mL}$ of ctDNA can be extracted in CSF instead of the $1 \mathrm{~mL}$ used in the present study, potentially improving the detection of small amounts of ctDNA. However, obtaining large amounts of CSF can be challenging and possibly harmful in these patients. The development of more sensitive methods, including next generation sequencing or more sensitive PCR methods, is necessary for the reliable detection of H3F3A K27M in DMG patients before the commencement of treatment.

\section{Conclusions}

In the present report, we found that the detection rate of $H 3 F 3 A \mathrm{~K} 27 \mathrm{M}$ by ddPCR in CSF obtained by lumbar puncture in DMG patients at presentation is low. Diagnosis by liquid biopsy at presentation may be possible in younger patients with a high protein concentration in CSF or evidence of leptomeningeal disease. Liquid biopsy is an exciting alternative to biopsy in DMGs, but both case selection and the development of more sensitive methods are vital.

Author Contributions: Conceptualization, J.O. and M.N.; methodology, J.O., J.W. and S.S.; pathological investigation, J.O. and A.K.; resources, Y.K., H.A., Y.T. and M.O. (Masayasu Okada); data curation, J.O. and J.W.; writing-original draft preparation, J.O. and M.N.; writing—review and editing, J.O., S.S., M.O. (Masayasu Okada), A.K. and M.N.; supervision, J.Y., M.O. (Makoto Oishi) and Y.F.; funding acquisition, M.N. and J.W. All authors have read and agreed to the published version of the manuscript.

Funding: This research was funded in part by grants from the Japan Society for Promotion of Science to M.N. (17K16632, 19K09476) and J.W. (19K18418) and from the 2016 Niigata University Interdisciplinary Research (U-go) grant to M.N.

Institutional Review Board Statement: The study was conducted according to the guidelines of the Declaration of Helsinki and approved by the Institutional Review Board of Niigata University Hospital (protocol code G2018-0008, approved 29 July 2018).

Informed Consent Statement: Informed consent was obtained from all subjects involved in the study.

Data Availability Statement: The data presented in this study are available upon reasonable request. 
Acknowledgments: The authors would like to acknowledge Osamu Onodera for shared usage of the ddPCR machine and Akihide Koyama of the Department of System Pathology for Neurological Disorders, Brain Research Institute, Niigata University, for the initial help with ddPCR.

Conflicts of Interest: The authors declare no conflict of interest.

\section{References}

1. Wu, G.; Diaz, A.K.; Paugh, B.S.; Rankin, S.L.; Ju, B.; Li, Y.; Zhu, X.; Qu, C.; Chen, X.; Zhang, J.; et al. The genomic landscape of diffuse intrinsic pontine glioma and pediatric non-brainstem high-grade glioma. Nat. Genet. 2014, 46, 444-450. [CrossRef] [PubMed]

2. $\quad$ Aihara, K.; Mukasa, A.; Gotoh, K.; Saito, K.; Nagae, G.; Tsuji, S.; Tatsuno, K.; Yamamoto, S.; Takayanagi, S.; Narita, Y.; et al. H3F3A K27M mutations in thalamic gliomas from young adult patients. Neuro Oncol. 2014, 16, 140-146. [CrossRef] [PubMed]

3. Castel, D.; Philippe, C.; Calmon, R.; Le Dret, L.; Truffaux, N.; Boddaert, N.; Pages, M.; Taylor, K.R.; Saulnier, P.; Lacroix, L.; et al. Histone H3F3A and HIST1H3B K27M mutations define two subgroups of diffuse intrinsic pontine gliomas with different prognosis and phenotypes. Acta Neuropathol. 2015, 130, 815-827. [CrossRef] [PubMed]

4. Khuong-Quang, D.A.; Buczkowicz, P.; Rakopoulos, P.; Liu, X.Y.; Fontebasso, A.M.; Bouffet, E.; Bartels, U.; Albrecht, S.; Schwartzentruber, J.; Letourneau, L.; et al. K27M mutation in histone H3.3 defines clinically and biologically distinct subgroups of pediatric diffuse intrinsic pontine gliomas. Acta Neuropathol. 2012, 124, 439-447. [CrossRef] [PubMed]

5. Mackay, A.; Burford, A.; Carvalho, D.; Izquierdo, E.; Fazal-Salom, J.; Taylor, K.R.; Bjerke, L.; Clarke, M.; Vinci, M.; Nandhabalan, M.; et al. Integrated Molecular Meta-Analysis of 1000 Pediatric High-Grade and Diffuse Intrinsic Pontine Glioma. Cancer Cell 2017, 32, 520-537. [CrossRef] [PubMed]

6. Taylor, I.C.; Hutt-Cabezas, M.; Brandt, W.D.; Kambhampati, M.; Nazarian, J.; Chang, H.T.; Warren, K.E.; Eberhart, C.G.; Raabe, E.H. Disrupting NOTCH slows diffuse intrinsic pontine glioma growth, enhances radiation sensitivity, and shows combinatorial efficacy with bromodomain inhibition. J. Neuropathol. Exp. Neurol. 2015, 74, 778-790. [CrossRef] [PubMed]

7. Louis, D.N.; Ohgaki, H.; Wiestler, O.D.; Cavenee, W.K.; Ellison, D.W.; Figarella-Branger, D.; Perry, A.; Reifenberger, G.; von Deimling, A. WHO Classification of Tumours of the Central Nervous System; IARC: Lyon, France, 2016.

8. Watanabe, J.; Natsumeda, M.; Okada, M.; Kobayashi, D.; Kanemaru, Y.; Tsukamoto, Y.; Oishi, M.; Kakita, A.; Fujii, Y. High detection rate of MYD88 mutations in cerebrospinal fluid from patients with central nervous system lymphomas. JCO Precis. Oncol. 2019, 3, 1-13. [CrossRef]

9. Huang, T.Y.; Piunti, A.; Lulla, R.R.; Qi, J.; Horbinski, C.M.; Tomita, T.; James, C.D.; Shilatifard, A.; Saratsis, A.M. Detection of Histone $\mathrm{H} 3$ mutations in cerebrospinal fluid-derived tumor DNA from children with diffuse midline glioma. Acta Neuropathol. Commun. 2017, 5, 28. [CrossRef] [PubMed]

10. Panditharatna, E.; Kilburn, L.B.; Aboian, M.S.; Kambhampati, M.; Gordish-Dressman, H.; Magge, S.N.; Gupta, N.; Myseros, J.S.; Hwang, E.I.; Kline, C.; et al. Clinically Relevant and Minimally Invasive Tumor Surveillance of Pediatric Diffuse Midline Gliomas Using Patient-Derived Liquid Biopsy. Clin. Cancer Res. 2018, 24, 5850-5859. [CrossRef] [PubMed]

11. Pan, C.; Diplas, B.H.; Chen, X.; Wu, Y.; Xiao, X.; Jiang, L.; Geng, Y.; Xu, C.; Sun, Y.; Zhang, P.; et al. Molecular profiling of tumors of the brainstem by sequencing of CSF-derived circulating tumor DNA. Acta Neuropathol. 2019, 137, 297-306. [CrossRef] [PubMed]

12. Miller, A.M.; Shah, R.H.; Pentsova, E.I.; Pourmaleki, M.; Briggs, S.; Distefano, N.; Zheng, Y.; Skakodub, A.; Mehta, S.A.; Campos, C.; et al. Tracking tumour evolution in glioma through liquid biopsies of cerebrospinal fluid. Nature 2019, 565, 654-658. [CrossRef] [PubMed]

13. Piccioni, D.E.; Achrol, A.S.; Kiedrowski, L.A.; Banks, K.C.; Boucher, N.; Barkhoudarian, G.; Kelly, D.F.; Juarez, T.; Lanman, R.B.; Raymond, V.M.; et al. Analysis of cell-free circulating tumor DNA in 419 patients with glioblastoma and other primary brain tumors. CNS Oncol. 2019, 8. [CrossRef] [PubMed]

14. Watanabe, J.; Natsumeda, M.; Kanemaru, Y.; Okada, M.; Oishi, M.; Kakita, A.; Fujii, Y. Comparison of circulating tumor DNA between body fluids in patients with primary central nervous system lymphoma. Leuk. Lymphoma 2019, 60, 3587-3589. [CrossRef] [PubMed]

15. Yamasaki, F.; Takayasu, T.; Nosaka, R.; Amatya, V.J.; Doskaliyev, A.; Akiyama, Y.; Tominaga, A.; Takeshima, Y.; Sugiyama, K.; Kurisu, K. Magnetic resonance spectroscopy detection of high lipid levels in intraaxial tumors without central necrosis: A characteristic of malignant lymphoma. J. Neurosurg. 2015, 122, 1370-1379. [CrossRef] [PubMed]

16. Abe, H.; Natsumeda, M.; Kanemaru, Y.; Watanabe, J.; Tsukamoto, Y.; Okada, M.; Yoshimura, J.; Oishi, M.; Fujii, Y. MGMT Expression Contributes to Temozolomide Resistance in H3K27M-Mutant Diffuse Midline Gliomas and MGMT Silencing to Temozolomide Sensitivity in IDH-Mutant Gliomas. Neurol. Med. Chir. 2018, 58, 290-295. [CrossRef] [PubMed]

17. Aoki, Y.; Hashizume, R.; Ozawa, T.; Banerjee, A.; Prados, M.; James, C.D.; Gupta, N. An experimental xenograft mouse model of diffuse pontine glioma designed for therapeutic testing. J. Neurooncol. 2012, 108, 29-35. [CrossRef] [PubMed]

18. Abe, H.; Natsumeda, M.; Okada, M.; Watanabe, J.; Tsukamoto, Y.; Kanemaru, Y.; Yoshimura, J.; Oishi, M.; Hashizume, R.; Kakita, A.; et al. MGMT Expression Contributes to Temozolomide Resistance in H3K27M-Mutant Diffuse Midline Gliomas. Front. Oncol. 2019, 9, 1568. [CrossRef] [PubMed]

19. Ko, C.; Kaushal, A.; Hammoud, D.A.; Steffen-Smith, E.A.; Bent, R.; Citrin, D.; Camphausen, K.; Warren, K.E. Role of early postradiation magnetic resonance imaging scans in children with diffuse intrinsic pontine glioma. Int. J. Radiat. Oncol. Biol. Phys. 2012, 83, 1252-1256. [CrossRef] [PubMed] 